\title{
Quantum Theory of Molecular Collisions in a Magnetic Field: Efficient Calculations Based on the Total Angular Momentum Representation
}

\section{Citation}

Tscherbul, Timur V. and Alexander Dalgarno. 2010. Quantum theory of molecular collisions in a magnetic field: Efficient calculations based on the total angular momentum representation. Journal of Chemical Physics 133(18): 184104.

\section{Published Version}

doi:10.1063/1.3503500

\section{Permanent link}

http://nrs.harvard.edu/urn-3:HUL.InstRepos:5141368

\section{Terms of Use}

This article was downloaded from Harvard University's DASH repository, and is made available under the terms and conditions applicable to Open Access Policy Articles, as set forth at http:// nrs.harvard.edu/urn-3:HUL.InstRepos:dash.current.terms-of-use\#OAP

\section{Share Your Story}

The Harvard community has made this article openly available.

Please share how this access benefits you. Submit a story.

\section{Accessibility}




\title{
Quantum theory of molecular collisions in a magnetic field: Efficient calculations based on the total angular momentum representation
}

\author{
T. V. Tscherbul ${ }^{\text {a) }}$ and A. Dalgarno \\ Harvard-MIT Center for Ultracold Atoms, Cambridge, Massachusetts 02138, USA and Institute for \\ Theoretical Atomic, Molecular and Optical Physics, Harvard-Smithsonian Center for Astrophysics, \\ Cambridge, Massachusetts 02138, USA
}

(Received 25 June 2010; accepted 28 September 2010; published online 9 November 2010)

\begin{abstract}
An efficient method is presented for rigorous quantum calculations of atom-molecule and molecule-molecule collisions in a magnetic field. The method is based on the expansion of the wave function of the collision complex in basis functions with well-defined total angular momentum in the body-fixed coordinate frame. We outline the general theory of the method for collisions of diatomic molecules in the ${ }^{2} \Sigma$ and ${ }^{3} \Sigma$ electronic states with structureless atoms and with unlike ${ }^{2} \Sigma$ and ${ }^{3} \Sigma$ molecules. The cross sections for elastic scattering and Zeeman relaxation in low-temperature collisions of $\mathrm{CaH}\left({ }^{2} \Sigma^{+}\right)$and $\mathrm{NH}\left({ }^{3} \Sigma^{-}\right)$molecules with ${ }^{3} \mathrm{He}$ atoms converge quickly with respect to the number of total angular momentum states included in the basis set, leading to a dramatic (>10-fold) enhancement in computational efficiency compared to the previously used methods [A. Volpi and J. L. Bohn, Phys. Rev. A 65, 052712 (2002); R. V. Krems and A. Dalgarno, J. Chem. Phys. 120, 2296 (2004)]. Our approach is thus well suited for theoretical studies of strongly anisotropic molecular collisions in the presence of external electromagnetic fields. (C) 2010 American Institute of Physics. [doi:10.1063/1.3503500]
\end{abstract}

\section{INTRODUCTION}

The advent of state-of-the-art experimental techniques for the production of cold molecular gases has opened new avenues of research into the mechanisms of molecular collisions and chemical reactions. ${ }^{1-3}$ Capable of delivering high-density samples of cold polar molecules, techniques such as buffer-gas cooling, ${ }^{4}$ Stark deceleration, ${ }^{5}$ and photoassociation ${ }^{6}$ provide direct access to a previously unexplored low-temperature regime, where the quantum nature of molecular collisions becomes important and external electromagnetic fields may be used to control chemical reactions. ${ }^{7,8}$ The possibility to fine-tune molecular interactions with external fields has far-reaching implications not only in chemical physics, but also precision spectroscopy, condensedmatter physics, and quantum information processing. ${ }^{1}$

Following the first observation of collisions between $\mathrm{CaH}$ molecules and He atoms in a magnetic trap, ${ }^{4}$ cold collision experiments have now been carried out with more than half a dozen molecular species including $\left.\mathrm{CaF}^{2} \Sigma^{+}\right),{ }^{9}$ $\mathrm{NH}\left({ }^{3} \Sigma^{-}\right)$and $\mathrm{ND}\left({ }^{3} \Sigma^{-}\right),{ }^{10} \mathrm{CrH}\left({ }^{6} \Sigma^{+}\right)$and $\mathrm{MnH}\left({ }^{7} \Sigma^{+}\right),{ }^{11}$ $\mathrm{OH}\left({ }^{2} \Pi\right),{ }^{12,13}$ and $\left.\mathrm{TiO}^{3} \Delta\right) .{ }^{14}$ Vibrationally inelastic collisions and chemical reactions of translationally cold $\mathrm{Cs}_{2}$ and $\mathrm{RbCs}$ molecules assembled by photo- and magnetoassociation from laser-cooled alkali-metal atoms were studied experimentally. ${ }^{15-17}$ Very recently, hyperfine relaxation in collisions of $\mathrm{KRb}\left({ }^{1} \Sigma^{+}\right)$molecules in the ground rovibrational state with $\mathrm{K}$ atoms and the chemical reaction $\mathrm{KRb}+\mathrm{KRb}$ $\rightarrow \mathrm{K}_{2}+\mathrm{Rb}_{2}$ were observed at a temperature of $250 \mathrm{nK}^{18}$

The need for a theoretical interpretation of these pioneering experiments stimulated the development of theoretical

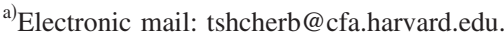

tools to elucidate the mechanisms of molecular collisions at low temperatures in the presence of external electromagnetic fields. Volpi and Bohn ${ }^{19}$ and Krems and Dalgarno ${ }^{20}$ were the first to develop a rigorous quantum mechanical formalism for atom-molecule and molecule-molecule collisions in the presence of external magnetic fields based on a fully uncoupled representation of the scattering wave function in the space-fixed (SF) coordinate frame. The fully uncoupled SF formalism was initially implemented to provide insight into the mechanisms of spin depolarization of ${ }^{2} \Sigma$ and ${ }^{3} \Sigma$ molecules induced by collisions with $\mathrm{He}$ atoms. ${ }^{21,22}$ More recently, several authors extended this formalism ${ }^{23}$ to study inelastic and reactive atom-molecule collisions in the presence of electric ${ }^{8,24,25}$ and microwave ${ }^{26}$ fields, moleculemolecule collisions in a magnetic field, ${ }^{27}$ collisions of molecular ions, ${ }^{28}$ and energy levels of van der Waals complexes. $^{29}$

A commonly stated disadvantage of the fully uncoupled $\mathrm{SF}$ formalism is that the dimension of the uncoupled basis grows dramatically as more rotational states and partial waves are added to it, thereby making scattering calculations on strongly anisotropic collision systems extremely challenging. ${ }^{30}$ A recent coupled-channel calculation of $\mathrm{O}_{2}\left({ }^{3} \Sigma_{g}^{-}\right)+\mathrm{O}_{2}\left({ }^{3} \Sigma_{g}^{-}\right)$collisions in a magnetic field included as many as 2526 uncoupled SF basis functions to produce converged cross sections at a collision energy of $0.1 \mathrm{~K}^{27}$ At higher collision energies more partial waves become involved and the fully uncoupled SF approach becomes computationally impractical. The same problem occurs in quantum reactive scattering calculations in the presence of external electromagnetic fields. ${ }^{8}$

In 1960 , Arthurs and Dalgarno ${ }^{31}$ pointed out that because 
the total angular momentum $J$ of the collision complex is conserved in the absence of external fields, the scattering problem can be factorized into smaller problems, which can be solved independently for each $J$. This factorization dramatically reduces the number of scattering channels, thereby facilitating quantum scattering calculations on molecular collisions to a great extent. External fields, however, break the isotropy of free space and induce couplings between different $J$. It is therefore not obvious whether the total angular momentum representation can be used to reduce the dimensionality of molecular collision problems in the presence of external fields.

In this work, we develop the theory of atom-molecule and molecule-molecule collisions in a magnetic field using the total angular momentum representation of Arthurs and Dalgarno. ${ }^{31}$ We apply our theory to calculate the cross sections for $\mathrm{CaH}\left({ }^{2} \Sigma\right)+\mathrm{He}$ and $\mathrm{NH}\left({ }^{3} \Sigma\right)+\mathrm{He}$ collisions, obtaining excellent agreement with the previous theoretical results $^{21,22}$ over a wide range of collision energies and magnetic fields. We find that the cross sections calculated at low collision energies converge quickly with increasing the number of total angular momentum states in the basis, leading to a substantial reduction in the number of scattering channels and more than a tenfold increase in computational efficiency.

The remainder of the paper is structured as follows. Section II A outlines the theory for diatomic molecules in the electronic states of ${ }^{2} \Sigma$ and ${ }^{3} \Sigma$ symmetry colliding with structureless particles. In Sec. II B, the theory is generalized to describe collisions of ${ }^{2} \Sigma$ and ${ }^{3} \Sigma$ molecules. Section III presents the numerical results demonstrating the feasibility and efficiency of our approach compared to those used earlier by other authors. ${ }^{19,20}$ A summary of the results and an outline of future research directions follow in Sec. IV.

\section{THEORY}

\section{A. Atom-molecule collisions}

In this section, we consider nonreactive collisions of diatomic molecules in the electronic states of ${ }^{2} \Sigma$ and ${ }^{3} \Sigma$ symmetry with structureless atoms (such as He or the alkalineearth atoms) in a magnetic field. The Hamiltonian for the atom-molecule system may be written as (in units of $\hbar$ )

$$
\hat{H}=-\frac{1}{2 \mu R} \frac{\partial^{2}}{\partial R^{2}} R+\frac{\hat{\ell}^{2}}{2 \mu R^{2}}+\hat{V}(\boldsymbol{R}, \boldsymbol{r})+\hat{H}_{\mathrm{as}},
$$

where $\boldsymbol{R}$ is the atom-molecule separation vector, $\mu$ is the reduced mass of the collision complex, $\hat{\ell}$ is the orbital angular momentum for the collision, and $\hat{V}(\boldsymbol{R}, \boldsymbol{r})$ is the atommolecule interaction potential. The asymptotic Hamiltonian $\hat{H}_{\text {as }}$ describes the rotational structure of a ${ }^{2} \Sigma$ molecule in its electronic and vibrational ground state in the presence of a uniform magnetic field $B,{ }^{20,32}$

$$
\hat{H}_{\mathrm{as}}=B_{e} \hat{N}^{2}+\gamma_{\mathrm{SR}} \hat{N} \cdot \hat{S}+2 \mu_{0} B \hat{S}_{Z}
$$

where $B_{e}$ is the rotational constant, $\hat{N}$ is the rotational angular momentum, $\hat{S}$ is the electron spin, $\hat{S}_{Z}$ is the projection of $\hat{S}$ on the magnetic field axis (which is the same as the quan- tization axis $Z$ of the SF frame), $\mu_{0}$ is the Bohr magneton, and $\gamma_{\mathrm{SR}}$ is a phenomenological spin-rotation constant. ${ }^{32}$ The rigid-rotor approximation used to derive Eq. (2) is valid if the vibrational frequency of the molecule is large compared to collision energy, which is true for low-temperature collisions considered in this work.

The asymptotic Hamiltonian for a ${ }^{3} \Sigma$ molecule may be obtained by adding to Eq. (2) a term describing the spin-spin interaction $^{32}$

$$
\frac{2}{3} \lambda_{\mathrm{SS}}\left(\frac{24 \pi}{5}\right)^{1 / 2} \sum_{q=-2}^{2}(-)^{q} Y_{2,-q}(\hat{r})[\hat{S} \otimes \hat{S}]_{q}^{(2)}
$$

where $\lambda_{S S}$ is the spin-spin constant and $[\hat{S} \otimes \hat{S}]_{q}^{(2)}$ is a spherical tensor product of $\hat{S}$ with itself.

We now introduce a body-fixed $(\mathrm{BF})$ coordinate frame. The $z$-axis of the BF frame is chosen to coincide with the vector $\boldsymbol{R}$ and the $y$-axis is chosen to be perpendicular to the collision plane. The orbital angular momentum $\hat{\ell}$ can be formally expressed via the total angular momentum of the collision complex as $\hat{\ell}=\hat{J}-\hat{N}-\hat{S}$ and the Hamiltonian (1) can be rewritten 30,33

$$
\hat{H}=-\frac{1}{2 \mu R} \frac{\partial^{2}}{\partial R^{2}} R+\frac{1}{2 \mu R^{2}}(\hat{J}-\hat{N}-\hat{S})^{2}+\hat{V}(R, \theta)+\hat{H}_{\text {as }},
$$

where $R=|\boldsymbol{R}|, r=|\boldsymbol{r}|$, and $\theta$ is the angle between $\boldsymbol{R}$ and $\boldsymbol{r}$. The BF Hamiltonian (4) is identical to its SF analog (1) except for the centrifugal term, which is now expressed in terms of the angular momentum operators $\hat{J}, \hat{N}$, and $\hat{S}$.

The wave function of the collision complex can be expanded in a direct product basis ${ }^{30,33}$

$$
\Psi=\frac{1}{R} \sum_{\alpha, J, \Omega} F_{\alpha J \Omega}^{M}(R)|\alpha\rangle|J M \Omega\rangle,
$$

where

$$
|\alpha\rangle=\left|N K_{N}\right\rangle|S \Sigma\rangle
$$

are the BF basis functions, $\Omega, K_{N}$, and $\Sigma$ are the projections of $\hat{J}, \hat{N}$, and $\hat{S}$ on the BF quantization axis $z\left(\Omega=K_{N}+\Sigma\right)$, and $M$ is the projection of $\hat{J}$ on the SF quantization axis $Z$. We note that $M$ is conserved for collisions in a magnetic field. The Wigner $D$-functions $|J M \Omega\rangle=\sqrt{(2 J+1) / 8 \pi^{2}}$ $\times D_{M \Omega}^{J \star}(\bar{\alpha}, \bar{\beta}, \bar{\gamma})$ depend on the Euler angles which specify the position of $\mathrm{BF}$ axes $x, y$, and $z$ in the $\mathrm{SF}$ frame. The functions $\left|N K_{N}\right\rangle=\sqrt{2 \pi} Y_{N K_{N}}(\theta, 0)$ and $|S \Sigma\rangle$ describe the rotational and spin degrees of freedom of the diatomic molecule in the $\mathrm{BF}$ frame. We note that the basis functions $|\alpha\rangle|J M \Omega\rangle$ with positive and negative $\Omega$ can be combined to form the eigenfunctions of the parity operator. Because inversion parity is conserved in the presence of a magnetic field, the scattering problem can be factorized into different parity blocks, leading to a reduction in the number of basis functions. We do not consider the factorization procedure in this work.

The radial expansion coefficients $F_{\alpha J \Omega}^{M}(R)$ satisfy a system of coupled-channel (CC) equations 


$$
\begin{aligned}
& {\left[\frac{d^{2}}{d R^{2}}+2 \mu E\right] F_{\alpha J \Omega}^{M}(R)} \\
& =2 \mu \sum_{\alpha^{\prime}, J^{\prime}, \Omega^{\prime}}\langle\alpha J \Omega| \hat{V}(R, \theta)+\frac{1}{2 \mu R^{2}}(\hat{J}-\hat{N}-\hat{S})^{2} \\
& \quad+\hat{H}_{\mathrm{as}}\left|\alpha^{\prime} J^{\prime} \Omega^{\prime}\right\rangle F_{\alpha^{\prime} J^{\prime} \Omega^{\prime}}^{M}(R),
\end{aligned}
$$

where $E$ is the total energy. The $\mathrm{CC}$ equations are parametrized by the matrix elements of the interaction potential, angular momentum coupling, and the asymptotic Hamiltonian, all of which can be evaluated in closed form as described below.

The atom-molecule interaction potential $V(\boldsymbol{R}, \boldsymbol{r})$ is a function of the atom-molecule separation $R$ and the Jacobi angle $\theta$, and can be expanded in Legendre polynomials

$$
V(R, \theta)=\sum_{\lambda} V_{\lambda}(R) P_{\lambda}(\cos \theta) .
$$

The Legendre polynomials depend on the same angle $\theta$ as the BF rotational functions $\left|N K_{N}\right\rangle$, so the angular integrals can be readily evaluated in terms of the radial expansion coefficients $V_{\lambda}(R)$ and $3-j$ symbols $\left(\begin{array}{c}\cdots \\ \cdots\end{array}\right)$ to give $\mathrm{e}^{30,33}$

$$
\begin{aligned}
\langle J M \Omega| & \left\langle N K_{N}\left|\left\langle S \Sigma|V(R, \theta)| J^{\prime} M^{\prime} \Omega^{\prime}\right\rangle\right| N^{\prime} K_{N}^{\prime}\right\rangle\left|S \Sigma^{\prime}\right\rangle \\
= & \delta_{J J^{\prime}} \delta_{M M^{\prime}} \delta_{\Sigma \Sigma^{\prime}} \times\left[(2 N+1)\left(2 N^{\prime}+1\right)\right]^{1 / 2} \\
& \times(-)^{K_{N}} \sum_{\lambda} V_{\lambda}(R)\left(\begin{array}{ccc}
N & \lambda & N^{\prime} \\
-K_{N} & 0 & K_{N}^{\prime}
\end{array}\right)\left(\begin{array}{ccc}
N & \lambda & N^{\prime} \\
0 & 0 & 0
\end{array}\right) .
\end{aligned}
$$

Because the interaction potential does not depend on the Euler angles and molecular spin variables, the matrix elements (9) are diagonal in $J, M, \Sigma$, and $K_{N}$ and independent of $J, M$, and $\Sigma$.

The rotational energy and the spin-rotation interaction are also independent of the Euler angles, so the matrix elements of the asymptotic Hamiltonian (2) can be simplified as follows:

$$
\begin{aligned}
& \left\langle\alpha\left|\left\langle J M \Omega\left|\hat{H}_{\mathrm{as}}\right| \alpha^{\prime}\right\rangle\right| J^{\prime} M^{\prime} \Omega^{\prime}\right\rangle \\
& =\delta_{J J^{\prime}} \delta_{M M^{\prime}} \delta_{\Omega \Omega^{\prime}}\left\langle\alpha\left|B_{e} \hat{N}^{2}+\gamma_{\mathrm{SR}} \hat{N} \cdot \hat{S}\right| \alpha^{\prime}\right\rangle \\
& +\left\langle\alpha\left|\left\langle J M \Omega\left|2 \mu_{0} B \hat{S}_{Z}\right| \alpha^{\prime}\right\rangle\right| J^{\prime} M^{\prime} \Omega^{\prime}\right\rangle .
\end{aligned}
$$

The first matrix element on the right-hand side can be readily evaluated by expressing the spin-rotation interaction via the shift operators ${ }^{34} \hat{N}_{ \pm}$and $\hat{S}_{ \pm}$,

$$
\begin{aligned}
\left\langle N K_{N}\left|\left\langle S \Sigma\left|B_{e} \hat{N}^{2}+\gamma_{\mathrm{SR}} \hat{N} \cdot \hat{S}\right| N^{\prime} K_{N}^{\prime}\right\rangle\right| S \Sigma^{\prime}\right\rangle \\
=\delta_{N N^{\prime}}\left[\left(B_{e} N(N+1)+\gamma_{\mathrm{SR}} K_{N} \Sigma\right) \delta_{K_{N} K_{N}^{\prime}} \delta_{\Sigma \Sigma^{\prime}}\right. \\
\quad+\frac{\gamma_{R}}{2}\left[N(N+1)-K_{N}^{\prime}\left(K_{N}^{\prime} \pm 1\right)\right]^{1 / 2} \\
\left.\quad \times\left[S(S+1)-\Sigma^{\prime}\left(\Sigma^{\prime} \mp 1\right)\right]^{1 / 2} \delta_{K_{N}, K_{N}^{\prime} \pm 1} \delta_{\Sigma, \Sigma^{\prime} \mp 1}\right] .
\end{aligned}
$$

The spin-rotation interaction couples the states with different $K_{N}$ and $\Sigma$, but conserves the sum $\Omega=K_{N}+\Sigma$.

In order to evaluate the matrix elements of the moleculefield interaction, we transform the operator $\hat{S}_{Z}$ in Eq. (2) to the BF frame

$$
\hat{S}_{Z}=\sum_{q} D_{0, q}^{1 \star}(\bar{\alpha}, \bar{\beta}, \bar{\gamma}) \hat{S}_{q}^{(1)},
$$

where the spherical tensor operators $\hat{S}_{q}^{(1)}$ are referred to the $\mathrm{BF}$ axes. Applying the Wigner-Eckart theorem to the matrix element $\left\langle S \Sigma\left|\hat{S}_{q}^{(1)}\right| S \Sigma^{\prime}\right\rangle$ and evaluating the angular integral over three $D$-functions, ${ }^{34}$ we find

$$
\begin{aligned}
\langle J M \Omega| & \left\langle N K_{N}\left|\left\langle S \Sigma\left|2 \mu_{0} B \hat{S}_{Z}\right| J^{\prime} M^{\prime} \Omega^{\prime}\right\rangle\right| N^{\prime} K_{N}^{\prime}\right\rangle\left|S \Sigma^{\prime}\right\rangle \\
= & 2 \mu_{0} B \delta_{N N^{\prime}} \delta_{K_{N} K_{N}^{\prime}}\left[(2 J+1)\left(2 J^{\prime}+1\right)\right]^{1 / 2} \\
& \times[(2 S+1) S(S+1)]^{1 / 2} \\
& \times \sum_{q}(-)^{M^{\prime}-\Omega^{\prime}-q+S-\Sigma}\left(\begin{array}{ccc}
J & 1 & J^{\prime} \\
M & 0 & -M^{\prime}
\end{array}\right) \\
& \times\left(\begin{array}{ccc}
J & 1 & J^{\prime} \\
\Omega & -q & -\Omega^{\prime}
\end{array}\right)\left(\begin{array}{ccc}
S & 1 & S \\
-\Sigma & q & \Sigma^{\prime}
\end{array}\right) .
\end{aligned}
$$

By summing Eqs. (11) and (13), we obtain the matrix elements of the asymptotic Hamiltonian in the BF basis. A few important properties of the matrix elements are worth noting: (i) the asymptotic Hamiltonian for a ${ }^{2} \Sigma$ molecule does not contain couplings between different $N$, (ii) the spin-spin interaction in ${ }^{3} \Sigma$ molecules [Eq. (3)] induces couplings between the ground $(N=0)$ and the second excited $(N=2)$ rotational states, (iii) an external magnetic field induces couplings between states of different $J$ and $\Omega$, and (iv) basis functions with the same $J$ and $\Omega$ but different $\Sigma$ are coupled by the spin-rotation (for $N>0$ ) and spin-spin interactions.

To evaluate the matrix elements of the orbital angular momentum in Eq. (4), we make use of the identity

$$
\begin{aligned}
(\hat{J}-\hat{N}-\hat{S})^{2}= & \hat{J}^{2}+\hat{N}^{2}+\hat{S}^{2}-2 \hat{J}_{z} \hat{N}_{z}-\hat{J}_{+} \hat{N}_{-}-\hat{J}_{-} \hat{N}_{+}-2 \hat{J}_{z} \hat{S}_{z} \\
& -\hat{J}_{+} \hat{S}_{-}-\hat{J}_{-} \hat{S}_{+}+2 \hat{N}_{z} \hat{S}_{z}+\hat{N}_{+} \hat{S}_{-}+\hat{N}_{-} \hat{S}_{+},
\end{aligned}
$$

where we have defined the shift operators $\hat{J}_{ \pm}=\hat{J}_{x} \pm i \hat{J}_{y}, \hat{N}_{ \pm}$ $=\hat{N}_{x} \pm i \hat{N}_{y}$, and $\hat{S}_{ \pm}=\hat{S}_{x} \pm i \hat{S}_{y}$. The BF components of $\hat{J}$ satisfy anomalous commutation relations, which we take into account by adopting the following convention ${ }^{34}$ for the matrix elements $\left\langle J M \Omega\left|\hat{J}_{ \pm}\right| J M \Omega^{\prime}\right\rangle=[J(J+1)$ $\left.-\Omega^{\prime}\left(\Omega^{\prime} \mp 1\right)\right]^{1 / 2} \delta_{\Omega, \Omega^{\prime} \mp 1}$. The matrix elements on the righthand side can be evaluated using the properties of shift operators $^{34}$ to yield 


$$
\begin{aligned}
\langle J M \Omega|\langle & \left.N K_{N}\left|\left\langle S \Sigma\left|(\hat{J}-\hat{N}-\hat{S})^{2}\right| J^{\prime} M^{\prime} \Omega^{\prime}\right\rangle\right| N^{\prime} K_{N}^{\prime}\right\rangle\left|S \Sigma^{\prime}\right\rangle \\
= & \delta_{J J^{\prime}} \delta_{M M^{\prime}} \delta_{N N^{\prime}}\left[\left\{J(J+1)+N(N+1)+S(S+1)-2 \Omega K_{N}-2 \Omega \Sigma+2 K_{N} \Sigma\right\} \delta_{\Omega \Omega^{\prime}} \delta_{K_{N} K_{N}^{\prime}} \delta_{\Sigma \Sigma^{\prime}}\right. \\
& -\left[J(J+1)-\Omega^{\prime}\left(\Omega^{\prime} \pm 1\right)\right]^{1 / 2}\left[N(N+1)-K_{N}^{\prime}\left(K_{N}^{\prime} \pm 1\right)\right]^{1 / 2} \delta_{\Omega, \Omega^{\prime} \pm 1} \delta_{K_{N}, K_{N}^{\prime} \pm 1} \delta_{\Sigma \Sigma^{\prime}} \\
& -\left[J(J+1)-\Omega^{\prime}\left(\Omega^{\prime} \pm 1\right)\right]^{1 / 2}\left[S(S+1)-\Sigma^{\prime}\left(\Sigma^{\prime} \pm 1\right)\right]^{1 / 2} \delta_{\Omega, \Omega^{\prime} \pm 1} \delta_{K_{N}, K_{N}^{\prime}} \delta_{\Sigma, \Sigma^{\prime} \pm 1} \\
& \left.+\left[N(N+1)-K_{N}^{\prime}\left(K_{N}^{\prime} \pm 1\right)\right]^{1 / 2}\left[S(S+1)-\Sigma^{\prime}\left(\Sigma^{\prime} \mp 1\right)\right]^{1 / 2} \delta_{\Omega \Omega^{\prime}} \delta_{K_{N}, K_{N}^{\prime} \pm 1} \delta_{\Sigma, \Sigma^{\prime} \mp 1}\right] .
\end{aligned}
$$

The BF basis functions with different $\Omega, K_{N}$, and $\Sigma$ are coupled by the off-diagonal Coriolis terms.

At large $R$, where the atom-molecule interaction potential is negligible compared with the collision energy, scattering boundary conditions may be applied to the radial expansion coefficients $F_{\alpha J \Omega}^{M}(R)$ in Eq. (5) to yield the $S$-matrix elements, which contain all the information about the dynamics of collision-induced transitions between different Zeeman states. To facilitate the application of the boundary conditions, it is convenient to transform the wave function to the SF basis

$$
|\beta \ell\rangle=\left|n_{\beta} J M \ell\right\rangle .
$$

The SF basis functions are the eigenfunctions of $\hat{\ell}^{2}$ with eigenvalues given by $\ell(\ell+1)$. In Eq. (16), $n_{\beta}$ stands for all other quantum numbers which depend on a particular angular momentum coupling scheme used. We emphasize that the SF basis defined by Eq. (16) is not the same as the fully uncoupled SF basis used by Volpi and Bohn ${ }^{19}$ and Krems and Dalgarno, ${ }^{20}$ but can be obtained from the latter by coupling the angular momenta $\hat{N}, \hat{S}$, and $\hat{\ell}$ to produce the resultant $\hat{J}$. The orthogonal transformation between the BF and SF representations may be written ${ }^{35}$

$$
|\beta \ell\rangle=\sum_{\alpha, \Omega} W_{\alpha J \Omega, \beta \ell}|\alpha J \Omega\rangle,
$$

where the transformation coefficients $W_{\alpha J \Omega, \beta \ell}$ form the matrix $\mathbf{W}$, which satisfies $\mathbf{W}^{T} \ell^{2} \mathbf{W}=\Lambda$, where $\ell^{2}$ is the matrix representation of $\hat{\ell}^{2}$ in the $\mathrm{BF}$ basis (15). The matrix $\mathbf{W}$ can be thought of as a collection of eigenvectors of $\ell^{2}$ arranged in columns, each corresponding to the diagonal matrix element of $\Lambda$ given by $\ell(\ell+1)$. We note that since $\ell^{2}$ (and hence $\mathbf{W}$ ) are diagonal in $J, M$, and $N$, these quantum numbers are not changed by the frame transformation (17).

As follows from Eq. (13), the asymptotic Hamiltonian (10) has off-diagonal matrix elements in the BF basis (6), which arise due to the spin-rotation interaction and external field couplings. In order to apply the asymptotic boundary conditions, we need to transform the wave function (5) to a representation in which the asymptotic Hamiltonian is diagonal $^{20}$

$$
|\gamma \ell\rangle=\sum_{\beta, \ell^{\prime}} C_{\beta \ell^{\prime}, \gamma \ell}\left|\beta \ell^{\prime}\right\rangle,
$$

where $\gamma$ labels the eigenvalues of $\mathbf{H}_{\mathrm{as}}$, and the transformation coefficients $C_{\beta \ell^{\prime}, \gamma \ell}$ correspond to the columns of the matrix $\mathbf{C}$, which satisfies $\mathbf{C}^{T} \mathbf{H}_{\mathrm{as}} \mathbf{C}$, where $\mathbf{H}_{\mathrm{as}}$ is the matrix of the asymptotic Hamiltonian in the SF basis (17). Because the asymptotic Hamiltonian (2) does not contain terms which couple basis functions with different $\ell$, we have $C_{\beta \ell^{\prime}, \gamma \ell}$ $=\delta_{\ell^{\prime} \ell} C_{\beta \gamma}$. The transformation (18) is similar to that described previously by Krems and Dalgarno. ${ }^{20}$ By combining Eqs. (17) and (18), we can express the asymptotic SF basis functions directly via the $\mathrm{BF}$ basis functions

$$
|\gamma \ell\rangle=\sum_{\beta, \ell^{\prime}} \sum_{\alpha, J, \Omega} W_{\alpha J \Omega, \beta \ell^{\prime}} C_{\beta \ell^{\prime}, \gamma \ell}|\alpha J \Omega\rangle .
$$

After transforming the wave function to the asymptotic SF basis (18) and applying the boundary conditions

$$
\begin{aligned}
F_{\gamma^{\prime} \ell^{\prime}}^{M}(R) \rightarrow & \delta_{\gamma \gamma^{\prime}} \delta_{\ell \ell^{\prime}} \exp \left[-i\left(k_{\gamma} R-\ell \pi / 2\right)\right] \\
& -\left(\frac{k_{\gamma}}{k_{\gamma^{\prime}}}\right)^{1 / 2} S_{\gamma \ell ; \gamma^{\prime} \ell^{\prime}}^{M} \exp \left[i\left(k_{\gamma^{\prime}} R-\ell^{\prime} \pi / 2\right)\right],
\end{aligned}
$$

we obtain the $S$-matrix elements or transition amplitudes between the Zeeman states $\gamma$ and $\gamma^{\prime}$. The integral cross sections can be evaluated from the $S$-matrix elements as ${ }^{20}$

$$
\sigma_{\gamma \rightarrow \gamma^{\prime}}=\frac{\pi}{k_{\gamma}^{2}} \sum_{\ell} \sum_{\ell, \ell^{\prime}}\left|\delta_{\ell \ell^{\prime}} \delta_{\gamma \gamma^{\prime}}-S_{\gamma \ell ; \gamma^{\prime} \ell^{\prime}}^{M}\right|^{2},
$$

where $k_{\gamma}^{2}=2 \mu\left(E-\epsilon_{\gamma}\right)=2 \mu E_{C}$ is the wave vector for the channel $\gamma$ and $E_{C}$ is the collision energy. As a result of applying the asymptotic transformations described above, the channel $\gamma$ has a well-defined asymptotic energy $\boldsymbol{\epsilon}_{\gamma}$.

The CC equations (7) were solved numerically on a radial grid extending from 2 to $100 a_{0}$ with a grid step of $0.04 a_{0}$ using the improved log-derivative method. ${ }^{36}$ The BF basis set included six rotational states of $\mathrm{CaH}\left(N_{\max }=5\right)$, five rotational states of $\mathrm{NH}\left(N_{\max }=4\right)$, and total angular momentum states up to $J_{\max }=7 / 2$ (for $\mathrm{CaH}+\mathrm{He}$ ) and $J_{\max }=4$ (for $\mathrm{NH}$ $+\mathrm{He}$ ). We used the same molecular constants and interaction 
potentials for $\mathrm{CaH}+\mathrm{He}$ and $\mathrm{NH}+\mathrm{He}$ as the previous theoretical studies. ${ }^{10,22,24}$ The resulting cross sections were converged to better than $10 \%$ for all magnetic fields and collision energies studied in this work. The accuracy of the calculated cross sections for collision energies below $0.01 \mathrm{~cm}^{-1}$ is limited by uncertainties in the $a b$ initio interaction potentials. ${ }^{21,22}$

\section{B. Molecule-molecule collisions}

The Hamiltonian for a nonreactive collision of two ${ }^{2} \Sigma$ molecules $A$ and $B$ may be written ${ }^{20,27,37,38}$

$$
\hat{H}=-\frac{1}{2 \mu R} \frac{\partial^{2}}{\partial R^{2}} R+\frac{\hat{\ell}^{2}}{2 \mu R^{2}}+\hat{V}\left(\boldsymbol{R}, \boldsymbol{r}_{A}, \boldsymbol{r}_{B}\right)+\hat{H}_{\mathrm{as}},
$$

where $\boldsymbol{R}$ is the molecule-molecule separation vector, $\hat{r}_{A}$ and $\hat{r}_{B}$ describe the orientation of molecules $A$ and $B$ in the SF frame, $\mu$ is the reduced mass of the collision complex, $\hat{\ell}$ is the orbital angular momentum for the collision, and $\hat{V}\left(R, \hat{r}_{A}, \hat{r}_{B}\right)$ is the interaction potential for two molecules frozen in their respective equilibrium geometries $\left(\boldsymbol{r}_{A}=\hat{r}_{A}\right.$ and $\boldsymbol{r}_{B}=\hat{r}_{B}$ ). The asymptotic Hamiltonian

$$
\hat{H}_{\mathrm{as}}=\hat{H}_{A}+\hat{H}_{B}
$$

describes noninteracting molecules in the presence of an external magnetic field and the Hamiltonians of individual molecules $A$ and $B$ are given by Eq. (2).

The orientation of vectors $\hat{r}_{A}$ and $\hat{r}_{B}$ in the BF frame (with $z$-axis along $\boldsymbol{R}$ ) is specified by the angles $\theta_{A}, \phi_{A}$ and $\theta_{B}, \phi_{B}$. The BF Hamiltonian takes the form

$$
\begin{aligned}
\hat{H}= & -\frac{1}{2 \mu R} \frac{\partial^{2}}{\partial R^{2}} R+\frac{1}{2 \mu R^{2}}\left(\hat{J}-\hat{N}_{A}-\hat{S}_{A}-\hat{N}_{B}-\hat{S}_{B}\right)^{2} \\
& +\hat{V}\left(R, \theta_{A}, \theta_{B}, \phi\right)+\hat{H}_{\text {as }} .
\end{aligned}
$$

Following the same strategy as described in Sec. II A, we expand the wave function of the molecule-molecule collision complex in the BF basis

$$
\Psi=\frac{1}{R} \sum_{\alpha_{A}, \alpha_{B}} \sum_{J, \Omega} F_{\alpha_{A} \alpha_{B} J \Omega}^{M}(R)\left|\alpha_{A}\right\rangle\left|\alpha_{B}\right\rangle|J M \Omega\rangle,
$$

where the internal basis functions

$$
\left|\alpha_{A}\right\rangle\left|\alpha_{B}\right\rangle=\left|N_{A} K_{N_{A}}\right\rangle\left|S_{A} \Sigma_{A}\right\rangle\left|N_{B} K_{N_{B}}\right\rangle\left|S_{B} \Sigma_{B}\right\rangle
$$

describe the rotational and spin degrees of freedom of two unlike molecules. The notation $\left|\alpha_{A}\right\rangle\left|\alpha_{B}\right\rangle$ implies that the basis function written first refers to molecule $A$ and the basis function written next refers to molecule $B$. For collisions of identical molecules, the basis set (26) should be modified to account for the effects of permutation symmetry. ${ }^{27}$ This can be accomplished by applying the symmetrization operator $1+\hat{P}_{A B}$ to the right-hand side of Eq. (25), where $\hat{P}_{A B}$ is the permutation operator defined by $\boldsymbol{r}_{A} \rightarrow \boldsymbol{r}_{B}, \quad \boldsymbol{r}_{B} \rightarrow \boldsymbol{r}_{A}$, $\boldsymbol{R} \rightarrow-\boldsymbol{R}^{20,39}$

Taking into account the orthogonality of basis functions $\left|\alpha_{A}\right\rangle$ and $\left|\alpha_{B}\right\rangle$, the matrix elements of the asymptotic Hamiltonian (23) can be separated in two contributions

$$
\begin{aligned}
& \left\langle\alpha_{A}\left|\left\langle\alpha_{B}\left|\left\langle J M \Omega\left|\hat{H}_{\mathrm{as}}\right| \alpha_{A}^{\prime}\right\rangle\right| \alpha_{B}^{\prime}\right\rangle\right| J^{\prime} M^{\prime} \Omega^{\prime}\right\rangle \\
& =\delta_{\alpha_{B} \alpha_{B}^{\prime}}\left\langle\alpha_{A}\left|\left\langle J M \Omega\left|\hat{H}_{A}\right| \alpha_{A}^{\prime}\right\rangle\right| J^{\prime} M^{\prime} \Omega^{\prime}\right\rangle \\
& \quad+\delta_{\alpha_{A} \alpha_{A}^{\prime}}\left\langle\alpha_{B}\left|\left\langle J M \Omega\left|\hat{H}_{\mathrm{B}}\right| \alpha_{B}^{\prime}\right\rangle\right| J^{\prime} M^{\prime} \Omega^{\prime}\right\rangle .
\end{aligned}
$$

The matrix elements of individual molecule Hamiltonians on the right-hand side can be evaluated following the procedures described in Sec. II A.

The spin-dependent interaction potential between the molecules can be expanded as

$$
\hat{V}\left(R, \theta_{A}, \theta_{B}, \phi\right)=\sum_{S, \Sigma} V_{S}\left(R, \theta_{A}, \theta_{B}, \phi\right)|S \Sigma\rangle\langle S \Sigma|,
$$

where $|S \Sigma\rangle$ are the spin functions, $\hat{S}=\hat{S}_{A}+\hat{S}_{B}$ is the total spin of the collision complex, and $\Sigma=\Sigma_{A}+\Sigma_{B}$ is its projection on the $z$-axis. The individual PESs $V_{S}\left(R, \theta_{A}, \theta_{B}, \phi\right)$ can be obtained as functions of BF angles $\theta_{A}$ and $\theta_{B}$ and $\phi=\phi_{A}-\phi_{B}$ from $a b$ initio calculations.

The BF scattering basis (26) is composed of direct products of spin functions for molecules $A$ and $B$, so it is convenient to re-express the spin-dependent interaction potential in terms of these functions. Using the identity

$$
\begin{aligned}
|S \Sigma\rangle= & \sum_{\Sigma_{A}, \Sigma_{B}}(-)^{S_{A}-S_{B}+\Sigma}(2 S+1)^{1 / 2}\left(\begin{array}{ccc}
S_{A} & S_{B} & S \\
\Sigma_{A} & \Sigma_{B} & -\Sigma
\end{array}\right) \\
& \times\left|S_{A} \Sigma_{A}\right\rangle\left|S_{B} \Sigma_{B}\right\rangle,
\end{aligned}
$$

we obtain an expansion equivalent to Eq. (28),

$$
\begin{aligned}
\hat{V}\left(R, \theta_{A}, \theta_{B}, \phi\right)= & \sum_{S, \Sigma} V_{S}\left(R, \theta_{A}, \theta_{B}, \phi\right) \\
& \times(-)^{2\left(S_{A}-S_{B}+\Sigma\right)} \sum_{\Sigma_{A}, \Sigma_{B} \Sigma_{A}^{\prime} \Sigma_{B}^{\prime}}(2 S+1) \\
& \times\left(\begin{array}{ccc}
S_{A} & S_{B} & S \\
\Sigma_{A} & \Sigma_{B} & -\Sigma
\end{array}\right)\left(\begin{array}{ccc}
S_{A} & S_{B} & S \\
\Sigma_{A}^{\prime} & \Sigma_{B}^{\prime} & -\Sigma
\end{array}\right) \\
& \times\left|S_{A} \Sigma_{A}\right\rangle\left|S_{B} \Sigma_{B}\right\rangle\left\langle S_{A} \Sigma_{A}^{\prime}\right|\left\langle S_{B} \Sigma_{B}^{\prime}\right| .
\end{aligned}
$$

The matrix elements of the interaction potential can now be evaluated in compact form

$$
\begin{aligned}
\left\langle\alpha_{A}\left|\left\langle\alpha_{B}|J M \Omega| \hat{V}\left(R, \theta_{A}, \theta_{B}, \phi\right) \mid \alpha_{A}^{\prime}\right\rangle\right| \alpha_{B}^{\prime}\right\rangle\left|J^{\prime} M^{\prime} \Omega^{\prime}\right\rangle \\
=\delta_{J J^{\prime}} \delta_{M M^{\prime}} \delta_{\Omega \Omega^{\prime}} \sum_{S, \Sigma}(-)^{2\left(S_{A}-S_{B}+\Sigma\right)}(2 S+1) \\
\quad \times\left(\begin{array}{ccc}
S_{A} & S_{B} & S \\
\Sigma_{A} & \Sigma_{B} & -\Sigma
\end{array}\right)\left(\begin{array}{ccc}
S_{A} & S_{B} & S \\
\Sigma_{A}^{\prime} & \Sigma_{B}^{\prime} & -\Sigma
\end{array}\right) \\
\quad \times\left\langle N_{A} K_{N_{A}}\left|\left\langle N_{B} K_{N_{B}}\left|V_{S}\left(R, \theta_{A}, \theta_{B}, \phi\right)\right| N_{A}^{\prime} K_{N_{A}}^{\prime}\right\rangle\right| N_{B}^{\prime} K_{N_{B}}^{\prime}\right\rangle .
\end{aligned}
$$

In order to evaluate the matrix element on the right-hand side, we expand the interaction potential in $\mathrm{BF}$ angular functions $27,37,38,40$ 


$$
V_{S}\left(R, \theta_{A}, \theta_{B}, \phi\right)=(4 \pi)^{3 / 2} \sum_{\lambda_{A}, \lambda_{B}, \lambda}\left(\frac{2 \lambda+1}{4 \pi}\right)^{1 / 2} V_{\lambda_{A} \lambda_{B} \lambda}^{S}(R) \sum_{m}\left(\begin{array}{ccc}
\lambda_{A} & \lambda_{B} & \lambda \\
m & -m & 0
\end{array}\right) Y_{\lambda_{A} m}\left(\theta_{A}, \phi_{A}\right) Y_{\lambda_{B},-m}\left(\theta_{B}, \phi_{B}\right),
$$

where $Y_{\lambda_{\nu} m}\left(\theta_{\nu}, \phi_{\nu}\right)$ are the spherical harmonics. The angular functions depend on the same angles as the BF basis functions (26), so the matrix element in Eq. (31) can be evaluated analytically ${ }^{34}$ to yield

$$
\begin{aligned}
\left\langle N_{A} K_{N_{A}}\left|\left\langle N_{B} K_{N_{B}}\left|V_{S}\left(R, \theta_{A}, \theta_{B}, \phi\right)\right| N_{A}^{\prime} K_{N_{A}}^{\prime}\right\rangle\right| N_{B}^{\prime} K_{N_{B}}^{\prime}\right\rangle & \\
= & {\left[\left(2 N_{A}+1\right)\left(2 N_{A}^{\prime}+1\right)\left(2 N_{B}+1\right)\left(2 N_{B}^{\prime}+1\right)\right]^{1 / 2} \sum_{\lambda_{A}, \lambda_{B}, \lambda} V_{\lambda_{A} \lambda_{B} \lambda}^{S}(R) \sum_{m}\left(\begin{array}{ccc}
\lambda_{A} & \lambda_{B} & \lambda \\
m & -m & 0
\end{array}\right) } \\
& \times\left[\left(2 \lambda_{A}+1\right)\left(2 \lambda_{B}+1\right)(2 \lambda+1)\right]^{1 / 2}(-)^{K_{N_{A}}+K_{N_{B}}}\left(\begin{array}{ccc}
N_{A} & \lambda_{A} & N_{A}^{\prime} \\
0 & 0 & 0
\end{array}\right)\left(\begin{array}{ccc}
N_{A} & \lambda_{A} & N_{A}^{\prime} \\
-K_{N_{A}} & m & K_{N_{A}}^{\prime}
\end{array}\right)\left(\begin{array}{ccc}
N_{B} & \lambda_{B} & N_{B}^{\prime} \\
0 & 0 & 0
\end{array}\right)\left(\begin{array}{ccc}
N_{B} & \lambda_{B} & N_{B}^{\prime} \\
-K_{N_{B}} & -m & K_{N_{B}}^{\prime}
\end{array}\right) .
\end{aligned}
$$

This expression in combination with Eq. (31) defines the matrix elements of the interaction potential for two distinguishable molecules. As in the atom-molecule case, the matrix elements are diagonal in $J, M$, and $\Omega$ and independent of $J$ and $M$. We note that Eqs. (31) and (33) are considerably simpler than the corresponding expressions arising in the SF formulation of the collision problem..$^{20,27}$

To complete the derivation of CC equations, we need to evaluate the matrix elements of the centrifugal kinetic energy in Eq. (22). This can be accomplished by expanding the orbital angular momentum $\hat{\ell}^{2}$ in terms of the ladder operators as described in Sec. II A. The final expression is only slightly more complicated than Eq. (15) and is presented in the Appendix.

The application of scattering boundary conditions for molecule-molecule collisions involves the same sequence of asymptotic transformations as described in Sec. II A for atom-molecule collisions. The first transformation uses the eigenvectors of $\ell^{2}$, whose matrix elements are presented in the Appendix. The second transformation requires the matrix C, which can be obtained by diagonalizing the matrix of the asymptotic Hamiltonian (23) in the SF basis.

Although in this work we focus on collisions of unlike molecules, the matrix elements for indistinguishable molecules can be obtained in the same way as described above using the definition of the symmetrized basis. ${ }^{39}$

\section{RESULTS}

In this section, we apply the methodology developed in Sec. II to evaluate the cross sections for elastic scattering and Zeeman relaxation in $\mathrm{CaH}+\mathrm{He}$ and $\mathrm{NH}+\mathrm{He}$ collisions in the presence of an external magnetic field. Low-temperature collisions of $\mathrm{CaH}\left({ }^{2} \Sigma\right)$ with ${ }^{3} \mathrm{He}$ have been studied both experimentally ${ }^{4}$ and theoretically using the fully uncoupled SF representation. ${ }^{21,25}$ Similarly, cold collisions of NH molecules with $\mathrm{He}$ atoms were the subject of several experimental and theoretical studies. ${ }^{10,22}$ We use these benchmark calculations below to verify our numerical results and gauge the efficiency of our theoretical approach.

\section{A. $\mathrm{CaH}\left({ }^{2} \Sigma^{+}\right)+\mathrm{He}$}

\section{Asymptotic energy levels}

An external magnetic field splits the ground rotational state of a ${ }^{2} \Sigma$ molecule like $\mathrm{CaH}$ into a doublet of Zeeman levels with $M_{S}= \pm 1 / 2$, where $M_{S}$ is the projection of the electron spin on the $Z$-axis. In the following, we will consider collisions of $\mathrm{He}$ atoms with $\mathrm{CaH}$ molecules initially in the low-field-seeking Zeeman sublevel $M_{S}=1 / 2$ of the ground rotational state (collisions of rotationally excited $\mathrm{CaD}$ molecules with $\mathrm{He}$ atoms in external fields were considered elsewhere). ${ }^{25}$ Figure 1 shows the lowest eigenvalues of the asymptotic Hamiltonian (2) as functions of the applied magnetic field for $M=1 / 2$. The eigenvalues corresponding to the upper and lower Zeeman levels of $\mathrm{CaH}$ have energies $\pm \mu_{0} B$. A third eigenvalue (shown in Fig. 1 by the red line) does not correspond to a physical Zeeman state.

The reason for the appearance of the unphysical eigenvalues is as follows. In the absence of external fields, the Hamiltonian matrix expressed in the basis (6) has a

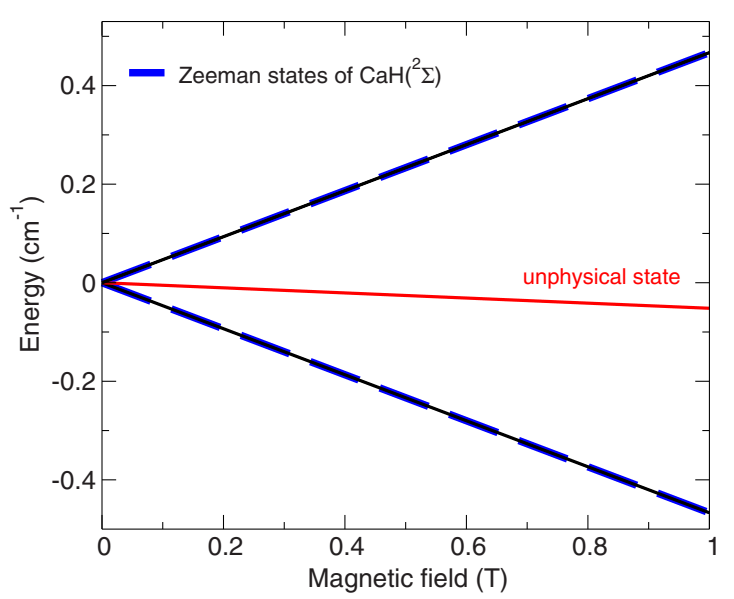

FIG. 1. Magnetic field dependence of the eigenvalues of the asymptotic Hamiltonian (2) is the BF basis with $N_{\max }=3$ and $J_{\max }=7 / 2$ (solid lines). Also shown are the Zeeman shifts of $\mathrm{CaH}$ in its $N=0$ rotational ground state (dashed lines). The unphysical state is shown by the red (light gray) line. 


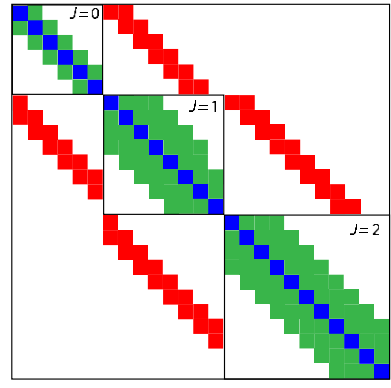

Total $J$ representation

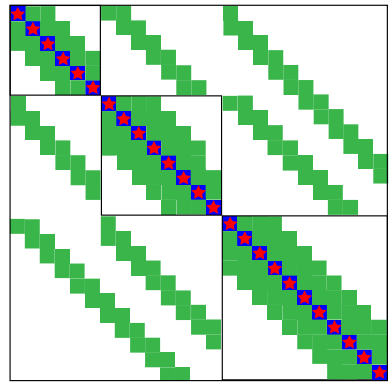

Uncoupled representation
FIG. 2. Schematic representation of the matrix of the asymptotic Hamiltonian. Blue squares denote diagonal matrix elements, green squares denote couplings induced by the anisotropy of the interaction potential, and red squares (left panel) and stars (right panel) denote couplings induced by an external magnetic field.

block-diagonal structure illustrated schematically in the left panel of Fig. 2. An external magnetic field induces couplings between the adjacent blocks with $J-J^{\prime}= \pm 1$. For a given $M$, the number of BF projections $\Omega$ increases with $J$ and so does the dimension of each $J$-block. When the Hamiltonian matrix is truncated at $J=J_{\max }$, the couplings between the $J_{\max }$ th and $\left(J_{\max }+1\right)$ th blocks drop out, altering the eigenvalues and eigenvectors of the asymptotic Hamiltonian and leading to the appearance of unphysical states.

Table I lists the first few eigenvalues and eigenvectors of the asymptotic Hamiltonian for $\mathrm{CaH}$ calculated in the $\mathrm{BF}$ basis with $N_{\max }=3$ and $J_{\max }=7 / 2$ for $M=1 / 2$. The physical states have the same energies as the Zeeman levels of $\mathrm{CaH}$ and their eigenvectors contain contributions from many values of $J$. In contrast, the eigenvector of the unphysical state shown in Fig. 1 is dominated by the largest value of $J$ included in the basis set $(J=7 / 2)$.

To further characterize the unphysical states, we consider the matrix of the asymptotic Hamiltonian in a truncated basis including only the ground rotational state of $\mathrm{CaH}\left({ }^{2} \Sigma^{+}\right)$ molecule $(N=0)$ augmented with two spin states ( $\Sigma$ $= \pm 1 / 2$ ) and two $J$-blocks with $J=1 / 2$ and $J=3 / 2$. Using Eqs. (11) and (13), we obtain a $4 \times 4$ matrix representation of the asymptotic Hamiltonian for $M=1 / 2$,

$$
\left(\begin{array}{cccc}
\delta & 2 \delta & -\sqrt{2} \delta & -\sqrt{2} \delta \\
2 \delta & \delta & \sqrt{2} \delta & \sqrt{2} \delta \\
-\sqrt{2} \delta & \sqrt{2} \delta & \frac{1}{5} \delta & \frac{4}{5} \delta \\
-\sqrt{2} \delta & \sqrt{2} \delta & \frac{4}{5} \delta & \frac{1}{5} \delta
\end{array}\right),
$$

where $\delta=\mu_{0} B / 3$ and the BF basis functions $|J \Omega\rangle$ labeling the columns of the matrix are arranged as follows (from left to right): $|1 / 2,-1 / 2\rangle,|1 / 2,1 / 2\rangle,|3 / 2,-1 / 2\rangle$, and $|3 / 2,1 / 2\rangle$. For $M=1 / 2, N=K_{N}=0$, and $\Sigma=\Omega$, so we can use the notation $|J \Omega\rangle$ as a shorthand for $|J M \Omega\rangle\left|N K_{N}\right\rangle|S \Sigma\rangle$, omitting the basis functions $\left|N K_{N}\right\rangle$ and $|S \Sigma\rangle$ for brevity. The matrix (34) can be diagonalized analytically to yield

$$
\begin{aligned}
& \lambda_{1}=-3 \delta=-\mu_{0} B, \\
& \lambda_{2}=-\frac{3}{5} \delta=-\frac{1}{5} \mu_{0} B, \\
& \lambda_{3}=\lambda_{4}=3 \delta=\mu_{0} B .
\end{aligned}
$$

The eigenvalues $\lambda_{1}, \lambda_{3}$, and $\lambda_{4}$ correspond to the $M_{S}$ $= \pm 1 / 2$ Zeeman states of $\mathrm{CaH}(N=0)$ with magnetic moments $\pm 1 \mu_{0}$ (see Fig. 1). The eigenvalue $\lambda_{2}$ has a magnetic moment of $-1 / 5 \mu_{0}$ and the corresponding eigenvector is given by

$$
|2\rangle=\frac{1}{\sqrt{2}}|J=3 / 2, \Omega=-1 / 2\rangle-\frac{1}{\sqrt{2}}|J=3 / 2, \Omega=1 / 2\rangle .
$$

Again, we see that the unphysical state is dominated by a single value of $J$, which is the larger of the two included in the basis set. This observation, together with the results listed in Table I, suggests that transitions to and from the unphysical states are strongly suppressed at low temperatures because of the long-range centrifugal barriers associated with the diagonal matrix elements of the Hamiltonian (15) between the basis functions with $J>0$.

TABLE I. The lowest eigenvalues (in units of $10^{-2} \mathrm{~cm}^{-1}$ ) and eigenvectors of the asymptotic Hamiltonian (2) calculated by numerical diagonalization of the matrix (10) in the BF basis with $N_{\max }=3$ and $J_{\max }=7 / 2$. The eigenvalues are arranged in the order of increasing energy. The eigenvectors are presented in terms of their expansion coefficients over the $|J \Omega\rangle$ basis with $N=0$ (see text for details). The unphysical eigenvalue shown in Fig. 1 by the red (light gray) line is highlighted in bold. The magnetic field is $0.1 \mathrm{~T}$.

\begin{tabular}{ccccccccc}
\hline \hline Energy & $\left|\frac{1}{2},-\frac{1}{2}\right\rangle$ & $\left|\frac{1}{2}, \frac{1}{2}\right\rangle$ & $\left|\frac{3}{2},-\frac{1}{2}\right\rangle$ & $\left|\frac{3}{2}, \frac{1}{2}\right\rangle$ & $\left|\frac{5}{2},-\frac{1}{2}\right\rangle$ & $\left|\frac{5}{2}, \frac{1}{2}\right\rangle$ & $\left|\frac{7}{2},-\frac{1}{2}\right\rangle$ & $\left|\frac{7}{2}, \frac{1}{2}\right\rangle$ \\
\hline-4.668623 & 0.577 & -0.577 & 0.414 & 0.403 & 0.005 & 0.005 & 0.0 & 0.0 \\
-4.668595 & 0.006 & -0.006 & -0.544 & 0.552 & -0.452 & -0.443 & -0.004 & -0.004 \\
-4.668572 & 0.0 & 0.0 & 0.005 & -0.005 & -0.531 & 0.538 & -0.463 & -0.463 \\
$-\mathbf{0 . 5 1 8 6 5 5}$ & $\mathbf{0 . 0}$ & $\mathbf{0 . 0}$ & $\mathbf{0 . 0}$ & $\mathbf{0 . 0}$ & $\mathbf{0 . 0}$ & $\mathbf{0 . 0}$ & $-\mathbf{0 . 7 0 7}$ & $\mathbf{0 . 7 0 7}$ \\
4.668656 & 0.732 & 0.679 & -0.038 & -0.037 & 0.0 & 0.0 & 0.0 & 0.0 \\
4.668674 & -0.361 & 0.454 & 0.582 & 0.570 & -0.008 & -0.008 & 0.0 & 0.0 \\
4.668697 & -0.006 & 0.006 & -0.439 & 0.455 & 0.552 & 0.543 & -0.005 & -0.005 \\
4.668718 & -0.0 & 0.0 & -0.004 & 0.004 & -0.458 & 0.468 & 0.535 & 0.535 \\
\hline \hline
\end{tabular}




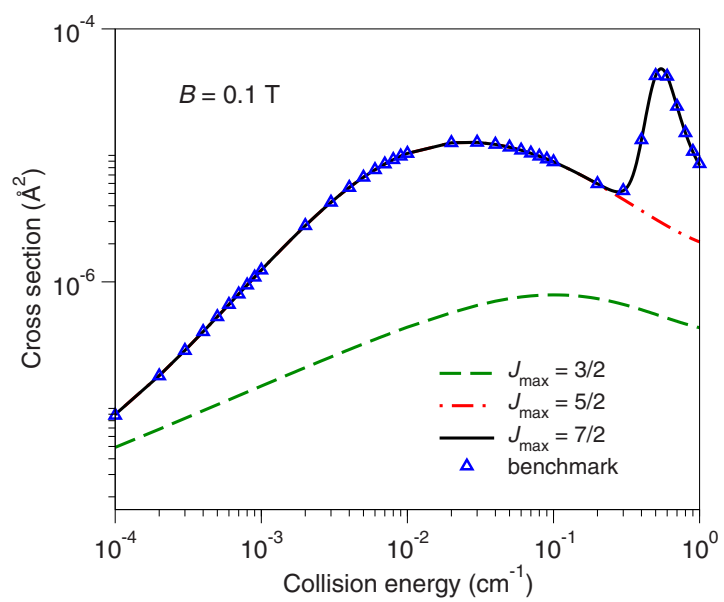

FIG. 3. Cross sections for Zeeman relaxation in $\mathrm{CaH}+\mathrm{He}$ collisions calculated as functions of collision energy using the BF representation for a magnetic field of $0.1 \mathrm{~T}$. The benchmark results obtained using the fully uncoupled SF representation are shown as symbols.

\section{Cross sections for Zeeman relaxation}

Figure 3 shows the cross sections for Zeeman relaxation in $\mathrm{CaH}+{ }^{3} \mathrm{He}$ collisions as functions of collision energy calculated with different numbers of $J$-states in the basis set (6). The cross sections calculated for $J_{\max }=7 / 2$ are in perfect agreement with the fully uncoupled SF results ${ }^{21}$ over the whole range of collision energies from $10^{-4}$ to $1 \mathrm{~cm}^{-1}$. The calculations employing the total angular momentum representation accurately reproduce both the broad maximum at $E_{C} \sim 2 \times 10^{-2} \mathrm{~cm}^{-1}$ and the $\ell=3$ shape resonance near $E_{C}$ $=0.5 \mathrm{~K}^{21}$. The cross sections for $J_{\max }=5 / 2$ agree well with the benchmark values at low collision energies, but do not reproduce the shape resonance near $0.5 \mathrm{~K}$. This might be due to an improper description of the $\ell=3$ centrifugal barrier in the entrance collision channel caused by the truncation of the basis set. The cross sections obtained in the calculation with $J_{\max }=3 / 2$ approach the benchmark values only at very low collision energies.

Table II compares the dimensions of scattering basis sets required to produce completely converged cross sections in the fully uncoupled SF and BF representations. The dimension of the uncoupled SF basis varies between 89 (for $M$ $=13 / 2$ ) and 315 (for $M=1 / 2$ ). The number of scattering channels required in the $\mathrm{BF}$ formalism is notably smaller, ranging from 48 (for $M=7 / 2$ ) to 140 (for $M=1 / 2$ ). Because the computational cost of coupled-channel calculations

TABLE II. The number of channels in fully uncoupled SF and BF basis sets for different $M$ and $J_{\max }$. Each basis set includes five rotational states of $\mathrm{CaH}\left({ }^{2} \Sigma^{+}\right)$. The ratio $\left(N_{\mathrm{SF}} / N_{\mathrm{BF}}\right)^{3}$ quantifies the extent to which the BF calculations are more computationally efficient. The ratio is calculated for $J_{\max }=7 / 2$.

\begin{tabular}{ccccc}
\hline \hline$|M|$ & Fully uncoupled SF & $\mathrm{BF}\left(J_{\max }=5 / 2\right)$ & $\mathrm{BF}\left(J_{\max }=7 / 2\right)$ & $\left(N_{\mathrm{SF}} / N_{\mathrm{BF}}\right)^{3}$ \\
\hline $1 / 2$ & 315 & 92 & 140 & 11.4 \\
$3 / 2$ & 297 & 74 & 122 & 14.4 \\
$5 / 2$ & 265 & 42 & 90 & 25.5 \\
$7 / 2$ & 223 & & 48 & 100.3 \\
$9 / 2$ & 176 & & & \\
\hline \hline
\end{tabular}

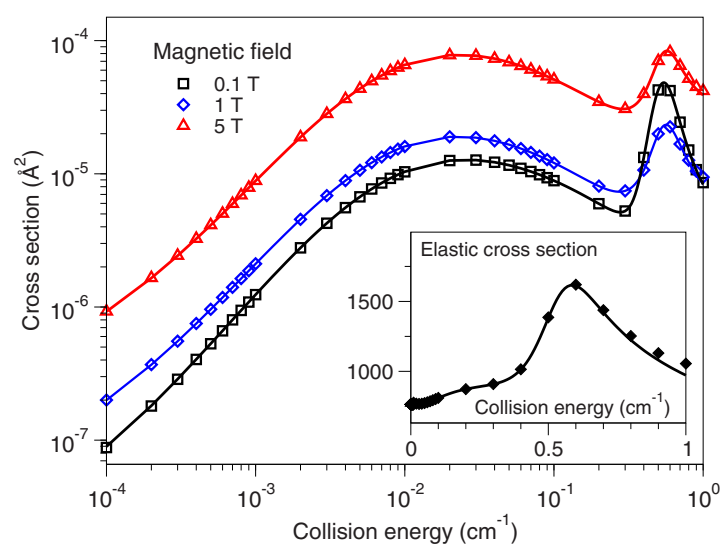

FIG. 4. Collision energy dependence of the cross sections for Zeeman relaxation in $\mathrm{CaH}+\mathrm{He}$ collisions calculated using the $\mathrm{BF}$ representation for magnetic fields of $0.1 \mathrm{~T}$ (lower trace), $1 \mathrm{~T}$ (middle trace), and $5 \mathrm{~T}$ (upper trace). The benchmark results obtained using the SF uncoupled representation are shown as symbols. The inset shows the elastic cross sections as functions of collision energy at a magnetic field of $0.1 \mathrm{~T}$.

scales as $N^{3}$ with the number of channels $N$, the BF approach is more than an order of magnitude more computationally efficient than its $\mathrm{SF}$ analog. ${ }^{19,20}$ The gain in computational efficiency is even larger at lower collision energies, where the BF method gives qualitative results with only three $J$-states in the basis set $\left(J_{\max }=5 / 2\right)$. As shown in Table II, the ratio of the efficiency of the BF and SF methods increases rapidly with $M$.

Figure 4 shows the cross sections for Zeeman relaxation in $\mathrm{CaH}+\mathrm{He}$ collisions calculated as functions of collision energy for different magnetic fields. The cross sections obtained using the present approach with $J_{\max }=7 / 2$ are in excellent agreement with fully uncoupled SF calculations for magnetic fields $0.1,1$, and $5 \mathrm{~T}$ and collision energies between $10^{-4}$ and $1 \mathrm{~cm}^{-1}$. The variation of the Zeeman relaxation cross sections with the applied magnetic field is plotted in Fig. 5 at a fixed collision energy of $10^{-3} \mathrm{~cm}^{-1}$. Our results are again in good agreement with the previous calculations, accurately reproducing the rapid increase of the cross section at low magnetic fields.

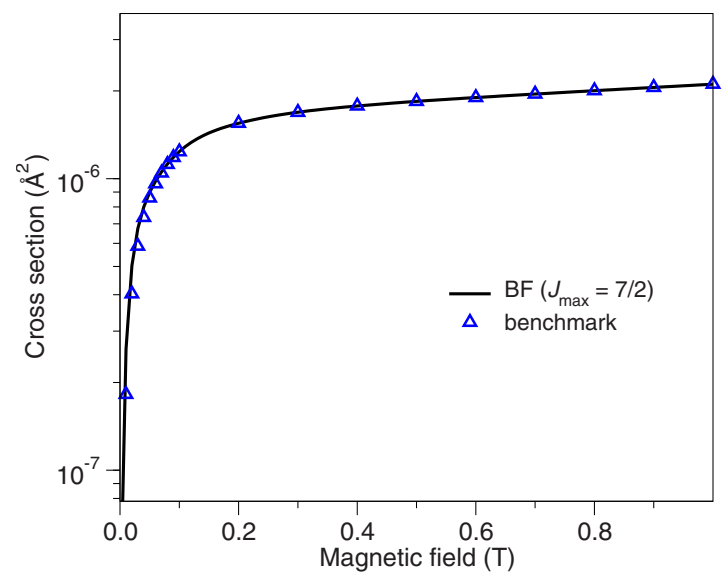

FIG. 5. Cross sections for Zeeman relaxation in $\mathrm{CaH}+\mathrm{He}$ collisions calculated using the BF representation (full line) as functions of magnetic field at a fixed collision energy of $0.001 \mathrm{~cm}^{-1}$. The benchmark results obtained using the fully uncoupled SF representation are shown as triangles. 


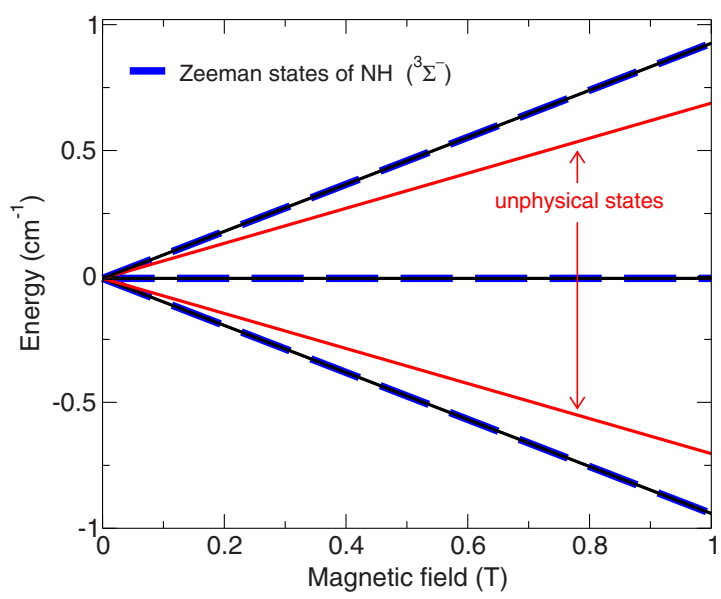

FIG. 6. Magnetic field dependence of the eigenvalues of the asymptotic Hamiltonian for $\mathrm{NH}\left({ }^{3} \Sigma\right)$ in the BF basis with $N_{\max }=4$ and $J_{\max }=4$ (solid lines). Also shown are the Zeeman shifts of $\left.\mathrm{NH}^{3}{ }^{3} \Sigma^{-}\right)$in its $N=0$ rotational ground state (dashed lines).

\section{B. $\mathrm{NH}\left({ }^{3} \Sigma^{-}\right)+\mathrm{He}$}

Figure 6 shows the magnetic field dependence of the eigenvalues of the asymptotic Hamiltonian for $\left.\mathrm{NH}^{(3} \Sigma^{-}\right)$for $M=0$. As in the case of $\mathrm{CaH}\left({ }^{2} \Sigma\right)+\mathrm{He}$ collisions considered above, we observe unphysical states arising from the truncation of the BF basis set at finite $J_{\max }$. The unphysical states in ${ }^{3} \Sigma$ molecules occur in pairs. The magnetic moments of the states within each pair are similar in magnitude but opposite in sign.

Figure 7 shows the cross section for Zeeman relaxation in collisions of $\mathrm{NH}$ molecules initially in the fully spinstretched state $M_{S}=1$ with ${ }^{3} \mathrm{He}$ atoms. The cross sections are summed over all final Zeeman states of $\mathrm{NH}$ and plotted as functions of collision energy. The triple peak around $0.5 \mathrm{~cm}^{-1}$ arises due to shape resonances in the incoming and outgoing collision channels. ${ }^{10}$ The BF calculations with $J_{\max }=4$ are in remarkable agreement with the benchmark results obtained using the SF uncoupled representation. ${ }^{10,22}$ At $E_{C}>0.1 \mathrm{~cm}^{-1}$, the agreement deteriorates with decreasing number of $J$ states in the basis set; in particular, the details of

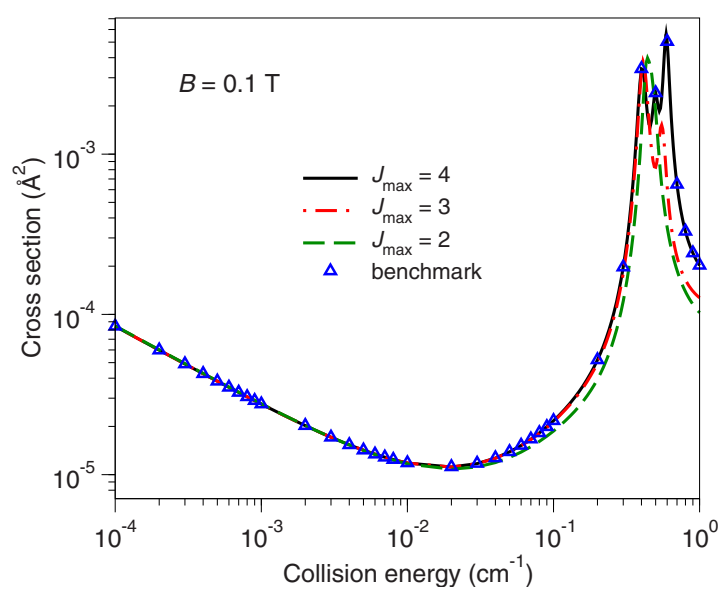

FIG. 7. Cross sections for Zeeman relaxation in $\mathrm{NH}+\mathrm{He}$ collisions calculated as functions of collision energy using the $\mathrm{BF}$ representation for a magnetic field of $0.1 \mathrm{~T}$. The benchmark results obtained using the fully uncoupled SF representation are shown by symbols. the resonant structure are not properly reproduced in $\mathrm{BF}$ calculations with $J_{\max } \leq 3$. Nevertheless, the agreement at $E_{C}$ $<0.01 \mathrm{~cm}^{-1}$ remains extraordinarily good even for the smallest BF basis with $J_{\max }=2$. Such a basis includes only 105 scattering channels for $M=0$, as opposed to the fully uncoupled SF basis, which includes 470 channels. The Wigner upturn in the dependence of the cross sections on collision energy that occurs at $E_{C} \sim 0.02 \mathrm{~cm}^{-1}$ is also accurately reproduced by the $\mathrm{BF}$ calculations.

\section{SUMMARY AND CONCLUSIONS}

We have developed an efficient method for rigorous quantum mechanical calculations of atom-molecule and molecule-molecule collisions in a magnetic field based on the expansion of the scattering wave function in basis functions with well-defined total angular momentum in the $\mathrm{BF}$ frame. We first outline the theory of the method for collisions of diatomic molecules in the ${ }^{2} \Sigma$ and ${ }^{3} \Sigma$ electronic states and then apply it to evaluate the cross sections for elastic energy transfer and inelastic Zeeman relaxation in low-temperature collisions of $\mathrm{CaH}\left({ }^{2} \Sigma\right)$ and $\mathrm{NH}\left({ }^{3} \Sigma\right)$ molecules with He atoms. Our calculated cross sections are in excellent agreement with the previous theoretical results ${ }^{20,22}$ over a wide range of collision energies and magnetic fields (Figs. 3-5 and 7).

The main advantage of our proposed approach lies in its fast convergence rate with respect to basis set size. As shown in Figs. 2 and 7, converged results at low collision energies can be obtained with only a few $J$-states in the basis set. The reason for this remarkably high convergence rate is as follows. In the total angular momentum representation, there are no couplings due to the interaction potential between the states of different $J$, so different $J$-blocks are only coupled by an external magnetic field (red squares in the left panel of Fig. 2). The long-range centrifugal barriers corresponding to BF basis states with $J>0$ suppress collisions at low kinetic energies, leading the cross sections to converge quickly with increasing $J$. By contrast, in the fully uncoupled SF representation, ${ }^{19,20}$ basis functions of different $\ell$ are coupled by the anisotropy of the interaction potential (green squares in the right panel of Fig. 2). The anisotropic couplings lower the height of the centrifugal barriers associated with $\ell>0$ basis states, making it necessary to include a large number of $\ell$-blocks to achieve convergence even at ultralow collision energies. ${ }^{41}$

The results presented in Table II demonstrate that quantum scattering calculations based on the total angular momentum representation in the $\mathrm{BF}$ frame are 10-50 times more computationally efficient than the previous calculations based on the fully uncoupled SF representation. ${ }^{19,20}$ Thus, our approach can be used to elucidate the effects of external electromagnetic fields on collisions of heavy atoms and molecules, which are often characterized by strongly anisotropic interaction potentials. Collisional properties of atommolecule and molecule-molecule mixtures are important in the context of current experimental work on sympathetic and evaporative cooling of molecules in external field traps. ${ }^{1-3}$

The Hamiltonian matrix in the total angular momentum representation has a block-diagonal structure in the absence 
of external fields. As shown in the left panel of Fig. 2, external fields induce couplings across different $J$-blocks, which leads to the appearance of unphysical eigenstates when the Hamiltonian matrix is truncated at a finite $J=J_{\max }$. We have shown that the unphysical states are characterized by the highest value of $J$ included in the basis set (Table I). As a result, the unphysical states do not matter for scattering calculations at low collision energies studied in this work (from $10^{-4}$ to $1 \mathrm{~cm}^{-1}$ ).

The BF formulation of collision problem presented in this work provides a meaningful starting point for the development of approximate methods of molecular collision theory. $^{30,33,42}$ In particular, it may be possible to neglect magnetic field couplings between certain $J$-blocks without compromising the accuracy of numerical results. Another way to simplify the scattering problem is to neglect the off-diagonal Coriolis couplings between different $\Omega$-states within the same $J$-block, thereby invoking the coupled states (CS) approximation. ${ }^{33,42}$ While the CS approximation allows for a substantial reduction of computational cost in coupledchannel calculations on low-temperature collisions of $\mathrm{Rb}$ atoms with $\mathrm{NH}_{3}$ molecules, ${ }^{43}$ and chemical reactions of $\mathrm{F}$ atoms with $\mathrm{HCl}$ molecules, ${ }^{44}$ it remains to be seen whether this approximation can provide accurate results for molecular collisions in the presence of external fields.

As our BF approach does not rely on a particular angular momentum coupling scheme, it should be generalizable to collisions and chemical reactions of complex molecules with many degrees of freedom, which have so far remained unexplored due to the enormity of coupled-channel basis sets required for convergence in the uncoupled SF formalism. In particular, the effects of hyperfine structure and internal angular momenta (such as the electronic orbital angular momentum in $\Pi$-state molecules or rotational motion in polyatomic molecules) can be easily incorporated into the $\mathrm{BF}$ formalism. By combining our approach with hyperspherical coordinate methods, ${ }^{8}$ it may be possible to create highly efficient hybrid techniques for elucidating the dynamics of chemical reactions $^{8,17,18}$ and three-body recombination ${ }^{45}$ in cold molecular gases in the presence of external electromagnetic fields.

\section{ACKNOWLEDGMENTS}

We thank Y. V. Suleimanov for helpful comments on the manuscript. This work was supported by the Chemical Science, Geoscience, and Bioscience Division of the Office of Basic Energy Science, Office of Science, U.S. Department of Energy and NSF grants to the Harvard-MIT Center for Ultracold Atoms and the Institute for Theoretical Atomic, Molecular and Optical Physics at Harvard University and the Smithsonian Astrophysical Observatory.

\section{APPENDIX: MATRIX ELEMENTS OF $\hat{\ell}^{2}$ FOR TWO DIATOMIC MOLECULES IN THE BF BASIS}

To evaluate the matrix elements of $\hat{\ell}^{2}$ in the BF basis, we first use the identity

$$
\begin{aligned}
\left(\hat{J}-\hat{N}_{A}-\hat{S}_{A}-\hat{N}_{B}-\hat{S}_{B}\right)^{2}= & \hat{J}^{2}+\hat{N}_{A}^{2}+\hat{S}_{A}^{2}+\hat{N}_{B}^{2}+\hat{S}_{B}^{2}-2 \hat{J}_{z} \hat{N}_{A_{z}}-\hat{J}_{+} \hat{N}_{A-}-\hat{J}_{-} \hat{N}_{A+}-2 \hat{J}_{z} \hat{S}_{A_{z}}-\hat{J}_{+} \hat{S}_{A-}-\hat{J}_{-} \hat{S}_{A+}-2 \hat{J}_{z} \hat{N}_{B_{z}}-\hat{J}_{+} \hat{N}_{B-} \\
& -\hat{J}_{-} \hat{N}_{B+}-2 \hat{J}_{z} \hat{S}_{B_{z}}-\hat{J}_{+} \hat{S}_{B-}-\hat{J}_{-} \hat{S}_{B+}+2 \hat{N}_{A_{z}} \hat{S}_{A_{z}}+\hat{N}_{A+} \hat{S}_{A-}+\hat{N}_{A-} \hat{S}_{A+}+2 \hat{N}_{A_{z}} \hat{S}_{B_{z}}+\hat{N}_{A+} \hat{S}_{B-}+\hat{N}_{A-} \hat{S}_{B+} \\
& +2 \hat{N}_{B_{z}} \hat{S}_{A_{z}}+\hat{N}_{B-} \hat{S}_{A+}+\hat{N}_{B+} \hat{S}_{A-}+2 \hat{N}_{B_{z}} \hat{S}_{B_{z}}+\hat{N}_{B-} \hat{S}_{B+}+\hat{N}_{B+} \hat{S}_{B-}+2 N_{A_{z}} \hat{N}_{B_{z}}+\hat{N}_{A+} \hat{N}_{B-}+\hat{N}_{A-} \hat{N}_{B+} \\
& +2 \hat{S}_{A_{z}} \hat{S}_{B_{z}}+\hat{S}_{A+} \hat{S}_{B-}+\hat{S}_{A-} \hat{S}_{B+}
\end{aligned}
$$

The matrix elements of all the 35 terms on the right-hand side can be evaluated using the properties of the shift operators. The final result is as follows:

$$
\begin{aligned}
& \left\langle J M \Omega\left|\left\langle\alpha_{A} \alpha_{B}\left|\left(\hat{J}-\hat{N}_{A}-\hat{S}_{A}-\hat{N}_{B}-\hat{S}_{B}\right)^{2}\right| J^{\prime} M^{\prime} \Omega^{\prime}\right\rangle\right| \alpha_{A}^{\prime} \alpha_{B}^{\prime}\right\rangle \\
& =\delta_{J J^{\prime}} \delta_{M M^{\prime}} \delta_{N_{A} N_{A}^{\prime}} \delta_{N_{B} N_{B}^{\prime}} \times\left[\left\{J(J+1)+N_{A}\left(N_{A}+1\right)+S_{A}\left(S_{A}+1\right)+N_{B}\left(N_{B}+1\right)+S_{B}\left(S_{B}+1\right)-2 \Omega K_{N_{A}}-2 \Omega \Sigma_{A}\right.\right. \\
& \left.-2 \Omega K_{N_{B}}-2 \Omega \Sigma_{B}+2 K_{N_{A}} \Sigma_{A}+2 K_{N_{A}} K_{N_{B}}+2 K_{N_{A}} \Sigma_{B}+2 \Sigma_{A} K_{N_{B}}+2 \Sigma_{A} \Sigma_{B}+2 K_{N_{B}} \Sigma_{B}\right\} \delta_{\Omega \Omega^{\prime}} \delta_{K_{N_{A}} K_{N_{A}}^{\prime}} \delta_{\Sigma_{A} \Sigma_{A}^{\prime}} \delta_{K_{N_{B}} K_{N_{B}}^{\prime}} \delta_{\Sigma_{B} \Sigma_{B}^{\prime}} \\
& -\left[J(J+1)-\Omega^{\prime}\left(\Omega^{\prime} \pm 1\right)\right]^{1 / 2}\left[N_{A}\left(N_{A}+1\right)-K_{N_{A}}^{\prime}\left(K_{N_{A}}^{\prime} \pm 1\right)\right]^{1 / 2} \delta_{\Omega, \Omega^{\prime} \pm 1} \delta_{K_{N_{A}}, K_{N_{A}}^{\prime} \pm 1} \delta_{\Sigma_{A} \Sigma_{A}^{\prime}} \delta_{K_{N_{B}} K_{N_{B}}^{\prime}} \delta_{\Sigma_{B} \Sigma_{B}^{\prime}} \\
& -\left[J(J+1)-\Omega^{\prime}\left(\Omega^{\prime} \pm 1\right)\right]^{1 / 2}\left[S_{A}\left(S_{A}+1\right)-\Sigma_{A}^{\prime}\left(\Sigma_{A}^{\prime} \pm 1\right)\right]^{1 / 2} \delta_{\Omega, \Omega^{\prime} \pm 1} \delta_{K_{N_{A}} K_{N_{A}}^{\prime}} \delta_{\Sigma_{A}, \Sigma_{A}^{\prime} \pm 1} \delta_{K_{N_{B}} K_{N_{B}}^{\prime}} \delta_{\Sigma_{B} \Sigma_{B}^{\prime}} \\
& -\left[J(J+1)-\Omega^{\prime}\left(\Omega^{\prime} \pm 1\right)\right]^{1 / 2}\left[N_{B}\left(N_{B}+1\right)-K_{N_{B}}^{\prime}\left(K_{N_{B}}^{\prime} \pm 1\right)\right]^{1 / 2} \delta_{\Omega, \Omega^{\prime} \pm 1} \delta_{K_{N_{A}} K_{N_{A}}^{\prime}} \delta_{\Sigma_{A} \Sigma_{A}^{\prime}} \delta_{K_{N_{B}}, K_{N_{B}}^{\prime} \pm 1} \delta_{\Sigma_{B} \Sigma_{B}^{\prime}} \\
& -\left[J(J+1)-\Omega^{\prime}\left(\Omega^{\prime} \pm 1\right)\right]^{1 / 2}\left[S_{B}\left(S_{B}+1\right)-\Sigma_{B}^{\prime}\left(\Sigma_{B}^{\prime} \pm 1\right)\right]^{1 / 2} \delta_{\Omega, \Omega^{\prime} \pm 1} \delta_{K_{N_{A}}} K_{N_{A}}^{\prime} \delta_{\Sigma_{A} \Sigma_{A}^{\prime}} \delta_{K_{N_{B}}} K_{N_{B}}^{\prime} \delta_{\Sigma_{B}, \Sigma_{B}^{\prime} \pm 1} \\
& +\left[N_{A}\left(N_{A}+1\right)-K_{N_{A}}^{\prime}\left(K_{N_{A}}^{\prime} \pm 1\right)\right]^{1 / 2}\left[N_{B}\left(N_{B}+1\right)-K_{N_{B}}^{\prime}\left(K_{N_{B}}^{\prime} \mp 1\right)\right]^{1 / 2} \delta_{\Omega \Omega^{\prime}} \delta_{K_{N_{A}}, K_{N_{A}}^{\prime} \pm 1} \delta_{\Sigma_{A} \Sigma_{A}^{\prime}} \delta_{K_{N_{B}}, K_{N_{B}}^{\prime} \mp 1} \delta_{\Sigma_{B}, \Sigma_{B}^{\prime}}
\end{aligned}
$$




$$
\begin{aligned}
& +\left[N_{A}\left(N_{A}+1\right)-K_{N_{A}}^{\prime}\left(K_{N_{A}}^{\prime} \pm 1\right)\right]^{1 / 2}\left[S_{A}\left(S_{A}+1\right)-\Sigma_{A}^{\prime}\left(\Sigma_{A}^{\prime} \mp 1\right)\right]^{1 / 2} \delta_{\Omega \Omega^{\prime}} \delta_{K_{N_{A}}, K_{N_{A}}^{\prime} \pm 1} \delta_{\Sigma_{A} \Sigma_{A \mp 1}^{\prime}} \delta_{K_{N_{B}}, K_{N_{B}}^{\prime}} \delta_{\Sigma_{B}, \Sigma_{B}^{\prime}} \\
& +\left[N_{A}\left(N_{A}+1\right)-K_{N_{A}}^{\prime}\left(K_{N_{A}}^{\prime} \pm 1\right)\right]^{1 / 2}\left[S_{B}\left(S_{B}+1\right)-\Sigma_{B}^{\prime}\left(\Sigma_{B}^{\prime} \mp 1\right)\right]^{1 / 2} \delta_{\Omega \Omega^{\prime}} \delta_{K_{N_{A}}, K_{N_{A}}^{\prime} \pm 1} \delta_{\Sigma_{A} \Sigma_{A}^{\prime}} \delta_{K_{N_{B}} K_{N_{B}}^{\prime}} \delta_{\Sigma_{B} \Sigma_{B}^{\prime} \mp 1} \\
& +\left[N_{B}\left(N_{B}+1\right)-K_{N_{B}}^{\prime}\left(K_{N_{B}}^{\prime} \pm 1\right)\right]^{1 / 2}\left[S_{A}\left(S_{A}+1\right)-\Sigma_{A}^{\prime}\left(\Sigma_{A}^{\prime} \mp 1\right)\right]^{1 / 2} \delta_{\Omega \Omega^{\prime}} \delta_{K_{N_{A}} K_{N_{A}}^{\prime}} \delta_{\Sigma_{A}, \Sigma_{A}^{\prime} \mp 1} \delta_{K_{N_{B}}, K_{N_{B}}^{\prime} \pm 1} \delta_{\Sigma_{B} \Sigma_{B}^{\prime}} \\
& +\left[N_{B}\left(N_{B}+1\right)-K_{N_{B}}^{\prime}\left(K_{N_{B}}^{\prime} \pm 1\right)\right]^{1 / 2}\left[S_{B}\left(S_{B}+1\right)-\Sigma_{B}^{\prime}\left(\Sigma_{B}^{\prime} \mp 1\right)\right]^{1 / 2} \delta_{\Omega \Omega^{\prime}} \delta_{K_{N_{A}} K_{N_{A}}^{\prime}} \delta_{\Sigma_{A} \Sigma_{A}^{\prime}} \delta_{K_{N_{B}}, K_{N_{B}}^{\prime} \pm 1} \delta_{\Sigma_{B} \Sigma_{B}^{\prime} \mp 1} \\
& \left.+\left[S_{A}\left(S_{A}+1\right)-\Sigma_{A}^{\prime}\left(\Sigma_{A}^{\prime} \pm 1\right)\right]^{1 / 2}\left[S_{B}\left(S_{B}+1\right)-\Sigma_{B}^{\prime}\left(\Sigma_{B}^{\prime} \mp 1\right)\right]^{1 / 2} \delta_{\Omega \Omega^{\prime}} \delta_{K_{N_{A}} K_{N_{A}}^{\prime}} \delta_{\Sigma_{A}, \Sigma_{A}^{\prime} \pm 1} \delta_{K_{N_{B}} K_{N_{B}}^{\prime}} \delta_{\Sigma_{B} \Sigma_{B}^{\prime} \mp 1}\right]
\end{aligned}
$$

${ }^{1}$ L. D. Carr, D. DeMille, R. V. Krems, and J. Ye, New J. Phys. 11, 055049 (2009).

${ }^{2}$ M. Schnell and G. Meijer, Angew. Chem., Int. Ed. 48, 6010 (2009).

${ }^{3}$ M. T. Bell and T. P. Softley, Mol. Phys. 107, 99 (2009).

${ }^{4}$ J. D. Weinstein, R. deCarvalho, T. Guillet, B. Friedrich, and J. M. Doyle, Nature (London) 395, 148 (1998).

${ }^{5}$ S. Y. T. van de Meerakker and G. Meijer, Faraday Discuss. 142, 113 (2009).

${ }^{6}$ O. Dulieu and C. Gabbanini, Rep. Prog. Phys. 72, 086401 (2009)

${ }^{7}$ R. V. Krems, Phys. Chem. Chem. Phys. 10, 4079 (2008).

${ }^{8}$ T. V. Tscherbul and R. V. Krems, J. Chem. Phys. 129, 034112 (2008).

${ }^{9}$ K. Maussang, D. Egorov, J. S. Helton, S. V. Nguyen, and J. M. Doyle, Phys. Rev. Lett. 94, 123002 (2005).

${ }^{10}$ W. C. Campbell, E. Tsikata, H.-I. Lu, L. D. van Buuren, and J. M. Doyle, Phys. Rev. Lett. 98, 213001 (2007); W. C. Campbell, T. V. Tscherbul, H.-I. Lu, E. Tsikata, R. V. Krems, and J. M. Doyle, ibid. 102, 013003 (2009).

${ }^{11}$ M. Stoll, J. M. Bakker, T. C. Steimle, G. Meijer, and A. Peters, Phys. Rev. A 78, 032707 (2008).

${ }^{12}$ J. J. Gilijamse, S. Hoekstra, S. Y. T. van de Meerakker, G. C. Groenenboom, and G. Meijer, Science 313, 1617 (2006).

${ }^{13}$ B. C. Sawyer, B. K. Stuhl, D. Wang, M. Yeo, and J. Ye, Phys. Rev. Lett. 101, 203203 (2008)

${ }^{14}$ M.-J. Lu and J. D. Weinstein, New J. Phys. 11, 055015 (2009).

${ }^{15}$ P. Staanum, S. D. Kraft, J. Lange, R. Wester, and M. Weidemüller, Phys. Rev. Lett. 96, 023201 (2006); N. Zahzam, T. Vogt, M. Mudrich, D Comparat, and P. Pillet, ibid. 96, 023202 (2006).

${ }^{16}$ E. R. Hudson, N. B. Gilfoy, S. Kotochigova, J. M. Sage, and D. DeMille, Phys. Rev. Lett. 100, 203201 (2008).

${ }^{17}$ S. Knoop, F. Ferlaino, M. Berninger, M. Mark, H.-C. Nägerl, R. Grimm, J. P. D'Incao, and B. D. Esry, Phys. Rev. Lett. 104, 053201 (2010).

${ }^{18}$ S. Ospelkaus, K.-K. Ni, D. Wang, M. H. G. de Miranda, B. Neyenhuis, G. Quéméner, P. S. Julienne, J. L. Bohn, D. S. Jin, and J. Ye, Science 327, 853 (2010).

${ }^{19}$ A. Volpi and J. L. Bohn, Phys. Rev. A 65, 052712 (2002).

${ }^{20}$ R. V. Krems and A. Dalgarno, J. Chem. Phys. 120, 2296 (2004).

${ }^{21}$ R. V. Krems, A. Dalgarno, N. Balakrishnan, and G. C. Groenenboom, Phys. Rev. A 67, 060703(R) (2003).

${ }^{22}$ R. V. Krems, H. R. Sadeghpour, A. Dalgarno, D. Zgid, J. Kłos, and G. Chałasiński, Phys. Rev. A 68, 051401(R) (2003); H. Cybulski, R. V.
Krems, H. R. Sadeghpour, A. Dalgarno, J. Kłos, G. C. Groenenboom, A. van der Avoird, D. Zgid, and G. Chałasiński, J. Chem. Phys. 122, 094307 (2005).

${ }^{23}$ R. V. Krems, in Proceedings of the Dalgarno Celebratory Symposium, edited by J. F. Babb, K. Kirby, and H. Sadeghpour (ICP, London, 2010), p. 281

${ }^{24}$ T. V. Tscherbul and R. V. Krems, Phys. Rev. Lett. 97, 083201 (2006).

${ }^{25}$ T. V. Tscherbul and R. V. Krems, J. Chem. Phys. 125, 194311 (2006).

${ }^{26}$ S. V. Alyabyshev, T. V. Tscherbul, and R. V. Krems, Phys. Rev. A 79, 060703(R) (2009); S. V. Alyabyshev and R. V. Krems, ibid. 80, 033419 (2009).

${ }^{27}$ T. V. Tscherbul, Yu. V. Suleimanov, V. Aquilanti, and R. V. Krems, New J. Phys. 11, 055021 (2009).

${ }^{28}$ G. Guillon, T. Stoecklin, and A. Voronin, Phys. Rev. A 77, 042718 (2008); G. Guillon and T. Stoecklin, J. Chem. Phys. 130, 144306 (2009).

${ }^{29}$ M. L. González-Martínez and J. M. Hutson, Phys. Rev. A 75, 022702 (2007).

${ }^{30}$ W. A. Lester, Jr., in Dynamics of Molecular Collisions, edited by W. H. Miller (Plenum, New York, 1976).

${ }^{31}$ A. M. Arthurs and A. Dalgarno, Proc. R. Soc. London, Ser. A 256, 540 (1960).

${ }^{32}$ M. Mizushima, The Theory of Rotating Diatomic Molecules (Wiley, New York, 1975)

${ }^{33}$ R. T. Pack, J. Chem. Phys. 60, 633 (1974).

${ }^{34}$ R. N. Zare, Angular Momentum (Wiley, New York, 1988).

${ }^{35}$ D. Skouteris, J. F. Castillo, and D. E. Manolopoulos, Comput. Phys. Commun. 133, 128 (2000).

${ }^{36}$ D. E. Manolopoulos, J. Chem. Phys. 85, 6425 (1986).

${ }^{37}$ S. Green, J. Chem. Phys. 62, 2271 (1975).

${ }^{38}$ G. C. Groenenboom, A. V. Fishchuk, and A. van der Avoird, J. Chem. Phys. 131, 124307 (2009).

${ }^{39}$ T. J. Heil, S. Green, and D. J. Kouri, J. Chem. Phys. 68, 2562 (1978).

${ }^{40}$ A. R. Offer, M. C. van Hemert, and E. F. van Dishoeck, J. Chem. Phys. 100, 362 (1994).

${ }^{41}$ V. Kokoouline, R. Santra, and C. H. Greene, Phys. Rev. Lett. 90, 253201 (2003); R. Santra and C. H. Greene, Phys. Rev. A 67, 062713 (2003).

${ }^{42}$ P. McGuire and D. J. Kouri, J. Chem. Phys. 60, 2488 (1974).

${ }^{43}$ P. S. Żuchowski and J. M. Hutson, Phys. Rev. A 79, 062708 (2009).

${ }^{44}$ G. Quéméner and N. Balakrishnan, J. Chem. Phys. 128, 224304 (2008).

${ }^{45}$ C. Ticknor and S. T. Rittenhouse, Phys. Rev. Lett. 105, 013201 (2010). 